\title{
Evaluación de la susceptibilidad de Aphis craccivora Koch (Hemiptera: Aphididae) a pirimicarb en condiciones de laboratorio
}

\author{
Evaluation of susceptibility of Aphis craccivora Koch (Hemiptera: Aphididae) \\ to pirimicarb in labotarory conditions
}

Víctor Tello ${ }^{1, *}$, Danny Canales ${ }^{2}$

\section{RESUMEN}

Se evaluó la susceptibilidad del pulgón negro de la alfalfa, Aphis craccivora (Koch) al ingrediente activo pirimicarb bajo condiciones de $26 \pm 2{ }^{\circ} \mathrm{C}, 60 \pm 15 \%$ de humedad relativa y un fotoperíodo de 12:12 (L:O). Los áfidos fueron colectados en dos localidades diferentes de la Región de Tarapacá, Chile, una con altas aplicaciones de insecticidas (Parca) y otra sin aplicación de estos (Noaza). Los insectos fueron expuestos a diferentes concentraciones de pirimicarb, aplicados con una torre Potter, determinando que el alto nivel de aplicaciones del insecticida Pirimor ${ }^{\circledR}$ (i.a. pirimicarb) para el control de áfidos en Parca ha generado una pérdida de la susceptibilidad a este producto observada en el bajo porcentaje de mortalidad (entre 40,40 $\pm 4,70 \%$ y 42,60 $\pm 4,35 \%$ ), cuando se aplicó la dosis mínima recomendada por el fabricante $\left(750,0 \mathrm{mg} \cdot \mathrm{L}^{-1}\right)$ comparado con la población susceptible que alcanzó mortalidades que variaron entre $77,40 \pm 3,31 \%$ y $87,30 \pm 3,35 \%$. El análisis probit no mostró diferencias significativas de la $\mathrm{CL}_{50} \mathrm{entre}$ las dos poblaciones estudiadas [Parca: $\mathrm{LC}_{50}=862,40 \mathrm{mgL}^{-1}(508,85-1.017,70)$; Noaza: $\left.\mathrm{LC}_{50}=519,14 \mathrm{mgL}^{-1}(369,07-615,06)\right]$, mientras que la proporción de resistencia de 1,7X, mostró que, las poblaciones de áfidos de la localidad de Parca aún no han desarrollado resistencia a pirimicarb.

Palabras clave: afidos, alfalfa, concentración letal, control químico.

\begin{abstract}
The susceptibility of cowpea aphid, Aphis craccivora (Koch) to active ingredient pirimicarb was evaluated under conditions of $26 \pm 2{ }^{\circ} \mathrm{C}, 60 \pm 15 \%$ RH and a photoperiod of 12:12 h(L:D). Aphids were collected in two different locations of the Región de Tarapacá, Chile, one with high applications of insecticides (Parca) and other without application (Noaza). Insects were exposed to different concentrations of pirimicarb applied with a Potter tower, determining that the high level of insecticide applications Pirimor ${ }^{\circledR}$ (a.i. pirimicarb) for controlling aphids in Parca, has generated a loss of susceptibility to this product due the low percentage of mortality (between $40.40 \pm 4.70 \%$ and $42.60 \pm 4.35 \%$ ) reached when the minimum dose recommended by manufacturer was applied $\left(750.0 \mathrm{mg} \cdot \mathrm{L}^{-1}\right)$ compared to the susceptible population which reached to mortalities ranging between $77.40 \pm 3.31 \%$ and $87.30 \pm 3.35 \%$. The probit analysis showed no significant differences between the $L C_{50}$ of two populations studied [Parca: $L C_{50}=862.40 \mathrm{mg} \mathrm{L}^{-1}$ (508.85-1017.70); Noaza: $L C_{50}=519.14 \mathrm{mg} \cdot \mathrm{L}^{-1}$ (369.07-615.06)], while the resistance ratio of 1.7X, showed that aphids population from Parca, have not yet developed resistance to pirimicarb.
\end{abstract}

Key words: aphids, alfalfa, lethal concentration, chemical control.

\section{Introducción}

El pulgón negro de la alfalfa, Aphis craccivora Koch (Hemiptera: Aphididae), se encuentra entre las 14 especies de áfidos de mayor importancia agrícola en el ámbito mundial
(Muñoz-Viveros et al., 2014). Este áfido es una especie cosmopolita, distribuyéndose en Chile desde la Región de Arica y Parinacota hasta la Región de Los Lagos, incluyendo la Isla de Pascua, sobre aproximadamente 54 especies vegetales como hortalizas, legumbres, frutales

\footnotetext{
Facultad de Recursos Naturales Renovables, Universidad Arturo Prat, Avenida Arturo Prat 2120, Iquique, Chile.

2 Fundación Chile, Avenida Parque Antonio Rabat Sur 6165, Vitacura, Santiago, Chile.

* Autor por correspondencia: victor.tello@unap.cl
}

Fecha de Recepción: 15 Noviembre, 2016.

Fecha de Aceptación: 7 Febrero, 2017.

DOI: $10.4067 /$ S0718-34292017005000011 
de pepita, frutales menores, oleaginosas, entre otras (Klein y Waterhouse, 2000).

El efecto más perjudicial de $A$. craccivora es la transmisión del virus del mosaico de las leguminosas (CABMV) que resulta en pérdida de rendimiento de los cultivos (De la Pava y Sepúlveda-Cano, 2015).

El control de pulgones incluyendo el de $A$. craccivora, se realiza comúnmente con plaguicidas sintéticos; sin embargo, el uso intensivo de insecticidas organofosforados y carbamatos ha generado un rápido desarrollo de resistencia a estos químicos (Kwon et al., 2009).

En la localidad de Parca (Región de Tarapacá, Chile) se ha realizado un uso indiscriminado del ingrediente activo (i.a.) pirimicarb, utilizado para el control de áfidos que atacan la alfalfa (Medicago sativa Linn.), no existe rotación de productos por lo tanto hay una gran posibilidad de que exista baja susceptibilidad al i.a. pirimicarb por parte de los áfidos del lugar.

Los carbamatos se han utilizado ampliamente en el control de plagas debido a su alta eficacia y baja persistencia. Pirimicarb (dimetilcarbamato de 2-dimetilamino-5,6-dimetilpirimidin-4-ilo) es un insecticida que se emplea extensivamente contra áfidos de frutales y hortalizas (Rongai et al., 1998; Furiatti y Lázarri, 2000; Araya et al., 2004; Walker et al., 2007; Raboudi et al., 2012; Fenoll et al., 2015). Presenta acción selectiva y baja toxicidad para los enemigos naturales y poco riesgo para las abejas (Araya et al., 2004). Bartlett (1963) clasificó a pirimicarb como un compuesto de clara selectividad para los coccinélidos. Hasta la fecha, el mecanismo molecular más conocido de la toxicidad del carbamato está representado por la inhibición reversible de la actividad de la colinesterasa (Lionetto et al., 2011). La acción tóxica de los carbamatos pueden implicar también otros mecanismos, incluyendo la sobreproducción de especies reactivas del oxígeno (Milatovic et al., 2006). El objetivo de esta investigación fue evaluar la susceptibilidad de dos poblaciones de $A$. craccivora al químico pirimicarb en condiciones de laboratorio.

\section{Materiales y Métodos}

\section{Obtención del pulgón negro de la alfalfa (Aphis craccivora)}

Los ejemplares de A. craccivora fueron colectados en dos localidades, Parca (20 $01^{\circ} 12,84^{\prime \prime}$ $\mathrm{S}, 69^{\circ} 12^{\prime} 24,46^{\prime}$ O O, $2.641 \mathrm{msnm}$ ) y Noaza
(1959'12,28” S, 6906'08,10" O, 3.288; msnm), ambas ubicadas en la Región de Tarapacá, Chile (desierto de Atacama). Los áfidos colectados en la primera localidad nunca fueron expuestos a insecticidas y los de la segunda localidad han estado expuestos constantemente a altas aplicaciones de pirimicarb. Los áfidos fueron colectados en bolsas Ziplock $®$, las que contenían papel absorbente en su interior, para evitar la condensación, provocada por la humedad del material vegetal en donde estaban ubicados los insectos. Las bolsas fueron rotuladas y colocadas en un contenedor con baja temperatura y trasladadas hacia el Laboratorio de Fitosanidad de la Estación Experimental Canchones (Universidad Arturo Prat), Región de Tarapacá, Chile (20²6'39,83" S, 69³2'00,76”' O, $995 \mathrm{msnm}$ ), donde se realizó su cultivo, así como la aplicación y evaluación de los tratamientos. Estas actividades se realizaron bajo condiciones ambientales de $26 \pm 2{ }^{\circ} \mathrm{C}, 60 \pm 15 \%$ de HR y un fotoperíodo de 12:12 h [luz (L): oscuridad (O)].

\section{Material vegetal}

Debido al pequeño tamaño de las hojas de alfalfa se sembraron semillas de poroto (Phaseolus vulgaris Linn. cultivar Canario), en siembra escalonada semanalmente, siendo regadas con una solución de $\mathrm{KNO}_{3}$ a 40 ppm de nitrógeno.

\section{Bioensayos de susceptibilidad de adultos de A. craccivora a pirimicarb}

Para este estudio se utilizaron diferentes concentraciones de pirimicarb (Pirimor ${ }^{\circledR} 500 \mathrm{~g} \cdot \mathrm{kg}^{-1}$ ) calculadas a partir de las dosis mínima (DRmín) y máxima (DRmáx) recomendadas por el fabricante para el control de A. craccivora en alfalfa que es de 150-250 g.ha ${ }^{-1}$ disueltos en $100 \mathrm{~L}$ de agua, equivalentes a 750,0 y $1.250,0 \mathrm{~g} \cdot \mathrm{L}^{-1}$ de pirimicarb, respectivamente. Se rociaron $2 \mathrm{~mL}$ de la solución de pirimicarb (Pirimor®) en cinco concentraciones calculada a partir de la dosis mínima recomendada: DRmín, DRmín+25\%DRmín, DRmín+50\%DRmín, DRmín+75\%DRmín y DRmín+100\%DRmín, correspondientes a 750,0; 937.5; 1.125,0; 1.312,5 y $1.500,0 \mathrm{mg} \cdot \mathrm{L}^{-1}$, respectivamente), además, de un testigo con agua destilada. Todos los tratamientos fueron aplicados mediante una Torre Potter (Makers Burkards Manufacturing Co. Ltd., Rickmansworth, U.K.) con una presión de $55 \mathrm{kPa}$ (Tello et al., 2013), 
sobre la base de una placa Petri de $12 \mathrm{~cm}$ de diámetro, la que contenía en su interior cinco discos de hojas de porotos (P. vulgaris), en cada disco fueron colocados 10 adultos ápteros (unidad experimental). Las placas se dejaron secar a temperatura ambiente $\left(26 \pm 2{ }^{\circ} \mathrm{C}\right.$, $60 \pm 15 \% \mathrm{RH}$ ) por una hora y posteriormente se taparon. Para las diluciones del insecticida y para el tratamiento control se utilizó agua destilada. El secado de las placas, una vez aplicadas las disoluciones, se realizó para prevenir la muerte por adherencia de los ejemplares a la superficie de la hoja (Iannacone y Lamas, 2003). Posteriormente fueron colocadas en cada caja de bioensayo. Las observaciones se realizaron cada 24 horas, por un tiempo total de 96 horas. Todas las observaciones fueron realizadas bajo lupa estereoscópica (Carl Zeiss, modelo Stemi SV6, Alemania) a 40x. Los áfidos adultos se consideraron muertos cuando fueron incapaces de moverse al ser estimulados suavemente con un pincel $\mathrm{N}^{\circ} 1$ de pelo de camello (Kraiss y Cullen, 2008). La unidad experimental fue un disco de hoja de $P$. vulgaris con 10 áfidos. Cada tratamiento fue repetido 10 veces. $\mathrm{La}$ mortalidad natural registrada en el testigo se utilizó para corregir la mortalidad por medio de la fórmula de Abbott (1925). Las poblaciones se consideraron significativamente diferentes cuando el IC al 95\% de las $\mathrm{CL}_{50,90}$ no se traslapaban con la población de referencia (Tello et al., 2013).

\section{Análisis estadístico}

El diseño experimental utilizado fue completamente aleatorizado. Se aplicaron análisis de varianzas y posteriormente la prueba de Tukey. Previo al análisis de varianzas, los porcentajes de mortalidad fueron normalizados mediante la transformación angular arcoseno $\sqrt{\mathrm{x} \otimes} \%^{-1}$. Para calcular las concentraciones letales $\left(\mathrm{CL}_{50}\right.$ y $\left.\mathrm{CL}_{90}\right)$, se aplicó un análisis probit, siguiendo la metodología aplicada por Tello et al. (2013). Las pendientes de las regresiones entre mortalidad (probit) y concentración $(\log )$. se obtuvieron utilizando el programa computacional SPSS Statistics 21. A partir de estos resultados se calculó la proporción de resistencia (PR) como el cociente entre la $\mathrm{CL}_{50}$ de la población sometida a presión de pirimicarb y la $\mathrm{CL}_{50}$ de la población susceptible, considerándose un valor mayor a $10 \mathrm{X}$ como desarrollo de resistencia (Cerna et al., 2009).

\section{Resultados y Discusión}

\section{Suceptibilidad de adultos de $A$. craccivora a pirimicarb}

Al evaluar el efecto de la concentración de pirimicarb sobre ejemplares adultos de $A$. craccivora, se encontró que después de 24 horas de haber sido aplicado el insecticida en forma directa sobre los áfidos provenientes de la localidad de Noaza, hubo diferencias significativas entre los tratamientos aplicados $\left(F_{(5,54)}=153,20 ; P<0,0001\right)$ (Tabla 1). Todas las concentraciones ensayadas arrojaron mortalidades diferentes al testigo. Las concentraciones $\geq 937,5 \mathrm{mg} \cdot \mathrm{L}^{-1}$ causaron porcentajes de mortalidad estadísticamente iguales,

Tabla 1. Mortalidad (\%) de adultos de Aphis craccivora a 24, 48, 72 and 96 h después de la aplicación de diferentes concentraciones de pirimicarb.

\begin{tabular}{|c|c|c|c|c|c|c|c|c|}
\hline \multirow{2}{*}{$\begin{array}{l}\text { Concentración } \\
\qquad\left(\mathrm{mg} \mathrm{L}^{-1}\right)\end{array}$} & \multicolumn{2}{|c|}{$24 \mathrm{~h}$} & \multicolumn{2}{|c|}{$48 \mathrm{~h}$} & \multicolumn{2}{|c|}{$72 \mathrm{~h}$} & \multicolumn{2}{|c|}{$96 \mathrm{~h}$} \\
\hline & Noaza & Parca & Noaza & Parca & Noaza & Parca & Noaza & Parca \\
\hline \multicolumn{9}{|c|}{$\%($ media $) \pm \mathrm{EE}^{3}$} \\
\hline $1.500,0$ & $98,80 \pm 1,20 \mathrm{a}^{1} \mathrm{~A}^{2}$ & $96,80 \pm 2,31$ a A & $100 \pm 0,00 \mathrm{aA}$ & $100,00 \pm 0,00 \mathrm{a} A$ & $100 \pm 0,00 \mathrm{a} A$ & $100,00 \pm 0,00 \mathrm{a} A$ & $100 \pm 0,00 \mathrm{a} A$ & $100,00 \pm 0,00 \mathrm{aA}$ \\
\hline $1.312,5$ & $96,40 \pm 2,56 \mathrm{a} \mathrm{A}$ & $75,90 \pm 6,57 \mathrm{~b} \mathrm{~B}$ & $100 \pm 0,00 \mathrm{aA}$ & $85,70 \pm 3,72$ b B & $100 \pm 0,00 \mathrm{a} A$ & $91,30 \pm 3,15 \mathrm{ab} \mathrm{B}$ & $100 \pm 0,00 \mathrm{a} A$ & $94,60 \pm 2,90 \mathrm{a} A$ \\
\hline $1.125,0$ & $95,40 \pm 1,89 \mathrm{a} \mathrm{A}$ & $71,70 \pm 4,70 \mathrm{bc} B$ & $100 \pm 0,00 \mathrm{aA}$ & $79,10 \pm 5,80 \mathrm{~b} \mathrm{~B}$ & $100 \pm 0,00 \mathrm{a} A$ & $79,10 \pm 5,80$ b B & $100 \pm 0,00 \mathrm{a} A$ & $79,10 \pm 5,80 \mathrm{~b} \mathrm{~B}$ \\
\hline 937,5 & $94,40 \pm 2,60 \mathrm{a} \mathrm{A}$ & $50,30 \pm 5,73 \mathrm{~cd} \mathrm{~B}$ & $100 \pm 0,00 \mathrm{aA}$ & $54,80 \pm 4,37 \mathrm{c} \mathrm{B}$ & $100 \pm 0,00$ a $\mathrm{A}$ & $54,80 \pm 4,37$ c B & $100 \pm 0,00 \mathrm{a} A$ & $54,80 \pm 4,37$ c B \\
\hline $750,0^{4}$ & $77,40 \pm 3,31 \mathrm{~b} \mathrm{~A}$ & $40,40 \pm 4,70 \mathrm{~d} \mathrm{~B}$ & $82,10 \pm 2,51 \mathrm{~b} \mathrm{~A}$ & $42,60 \pm 4,35$ c B & $85,30 \pm 3,75 \mathrm{bA}$ & $42,60 \pm 4,35 \mathrm{c} \mathrm{B}$ & $87,30 \pm 3,35 \mathrm{bA}$ & $42,60 \pm 4,35 \mathrm{c} \mathrm{B}$ \\
\hline 0.0 & $0,00 \pm 0,00 \mathrm{c} \mathrm{A}$ & $0,00 \pm 0,00 \mathrm{e} A$ & $0,00 \pm 0,00 \mathrm{c} \mathrm{A}$ & $0,00 \pm 0,00 \mathrm{dA}$ & $0,00 \pm 0,00 \mathrm{cA}$ & $0,00 \pm 0,00 \mathrm{~d} \mathrm{~A}$ & $0,00 \pm 0,00 \mathrm{c} \mathrm{A}$ & $0,00 \pm 0,00 \mathrm{dA}$ \\
\hline
\end{tabular}

1 Medias seguidas por la misma letra mayúscula en cada columna no difieren significativamente de acuerdo a la Prueba de Tukey al 5\% de probabilidad.

2 Medias seguidas por la misma letra minúscula en cada fila, dentro de cada tiempo de evaluación, no difieren significativamente de acuerdo a la prueba t-Student al 5\% de probabilidad.

3 Error estándar.

4 Dosis mínima recomendada. 
variando entre $94,40 \%$ y $98,80 \%$. El tratamiento que contenía la DRmín presentó un 77,40ะ3,31\% de mortalidad. A las 48 horas, se repitió el mismo esquema anterior con diferencias significativas entre los tratamientos $\left(F_{(5,54)}=2.203,61 ; P<0,0001\right)$. El tratamiento DRmín produjo $82,10 \pm 2,51 \%$ de mortalidad y las concentraciones $\geq 937,5 \mathrm{mg} \cdot \mathrm{L}^{-1}$ alcanzaron $100 \%$ de mortalidad. Estos resultados se repitieron a las $72 \mathrm{~h}\left(F_{(5,54)}=409,57 ; P<0,0001\right)$ y $96 \mathrm{~h}\left(F_{(5,54)}=472,61 ; P<0,0001\right)$ después de haber aplicado los tratamientos.

La aplicación de pirimicarb a la población proveniente de la localidad de Parca mostró diferencias significativas a las 24, 48, 72 y 96 horas después de haberse aplicado los tratamientos $(24 \mathrm{~h}$ : $F_{(5,54)}=65,92, P<0,0001 ; 48 \mathrm{~h}: F_{(5,54)}=94,83$, $P<0,0001 ; 72 \mathrm{~h}: F_{(5,54)}=101,84, P<0,0001$ y 96h: $\left.F_{(5,54)}=111,28, P<0,0001\right)($ Tabla 1). La DRmín presentó una mortalidad que varió entre el $40,40 \%$ y $42,60 \%$. Mortalidades sobre el $75 \%$ fueron alcanzadas con concentraciones de pirimicarb entre $1.125,0$ y $1.500,0 \mathrm{mg} \cdot \mathrm{L}^{-1}$ a partir de las 48 $\mathrm{h}$ después de aplicados a los tratamientos.

Al comparar la mortalidad entre las dos poblaciones del áfido se encontraron diferencias significativas para la mayoría de las concentraciones evaluadas a las 24 h, 48 h, 72 h y 96 h. Donde no se encontraron diferencias fue a la concentración mayor $\left(1.500 \mathrm{mgL}^{-1}\right)$ en todos los períodos de evaluación y para la concentración de 1.312,5 $\mathrm{mg} \cdot \mathrm{L}^{-1}$ a las $96 \mathrm{~h}$ de haberse aplicado el tratamiento.

En la Tabla 2 se muestran los resultados del análisis probit sobre los datos de concentraciónmortalidad para dos poblaciones de A. craccivora tratadas con pirimicarb. Los valores de concentraciones letales para las dos poblaciones de A. craccivora, observados después de 24 horas de haberse aplicado el insecticida muestran que solo en la $\mathrm{CL}_{90}$ hubo diferencias significativas. Aun cuando la $\mathrm{CL}_{50}$ para ambas poblaciones no presentaron diferencias significativas, para el caso de los áfidos de Noaza las $\mathrm{CL}_{50,90}$ están dentro del rango de lo recomendado por el fabricante (DRmín $<\mathrm{CL}_{50,90}<$ DRmáx). En cambio para los áfidos de la localidad de Parca, sólo la $\mathrm{CL}_{50}$ se ubicó entre las dosis mínima y máxima recomendadas por el fabricante (DRmín $\left.<\mathrm{CL}_{50}=826,40<\mathrm{DRmáx}\right)$. En cambio la $\mathrm{CL}_{90}$, para las poblaciones de estos áfidos, supera la DRmáx. A partir de estos resultados se calculó la proporción de resistencia la que fue de $1,7 \mathrm{X}$.

Rizk et al. (2014) aplicaron pirimicarb a distintas poblaciones de Aphis gossypii Glover (Hemiptera: Aphididae) obteniendo valores de $\mathrm{CL}_{50}$ entre 328,47 y $482,22 \mathrm{mg} \cdot \mathrm{L}^{-1}$. Herron et al. (2001) determinaron la $\mathrm{CL}_{50}$ para poblaciones resistentes y susceptibles de A. gossypii a pirimicarb la que varió entre $1.727,0 \mathrm{y}$ $4.909,0 \mathrm{mg} \cdot \mathrm{L}^{-1}$ y 1,15 y $1,52 \mathrm{mg} \cdot \mathrm{L}^{-1}$, respectivamente. Tabacian et al. (2011) obtuvieron una $\mathrm{CL}_{50}$ de 688 y $1.427 \mathrm{mg} \cdot \mathrm{L}^{-1}$ de pirimicarb para dos poblaciones de A. gossypii. En un estudio realizado en Chile, Fuentes-Contreras et al. (2013) encontraron una gran variación en la mortalidad causada por pirimicarb con $\mathrm{CL}_{50}$ fluctuando desde $7,34 \mathrm{mg} \cdot \mathrm{L}^{-1}$ hasta 407,45 $\mathrm{mg} \cdot \mathrm{L}^{-1}$ en Myzus persicae (Sulzer) (Hemiptera: Aphididae). En ese estudio utilizaron dos genotipos como referentes de resistencia y susceptibilidad (testigos), línea 4824J procedente de Francia y 4255A procedente de Inglaterra, respectivamente. La línea resistente presentó una $\mathrm{LC}_{50}=26,90 \mathrm{mg} \cdot \mathrm{L}^{-1}$ y la línea susceptible una $\mathrm{LC}_{5}=10,38 \mathrm{mg} \cdot \mathrm{L}^{-1}$. Baker (1978) encontró resistencia de $M$. persicae a los ingredienes activos $\left(\mathrm{mg} \cdot \mathrm{L}^{-1}\right)$ maldison $\left(\mathrm{CL}_{50}=192,00\right)$, demetonS-metil $\left(\mathrm{CL}_{50}=76,40\right)$, mevinfos $\left(\mathrm{CL}_{50}=167,00\right)$, acefate $\left(\mathrm{CL}_{50}=7,84\right)$ y naled $\left(\mathrm{CL}_{50}=66,30\right)$, pero no a pirimicarb $\left(\mathrm{CL}_{50}=0,55\right)$. Al contrario, autores

Tabla 2. Concentración letal $\left(\mathrm{CL}_{50,90} \mathrm{en} \mathrm{mg} \cdot \mathrm{L}^{-1}\right)$ de pirimicarb, estimada por análisis probit, de dos poblaciones de Aphis craccivora.

\begin{tabular}{|c|c|c|c|c|c|c|c|}
\hline \multirow{2}{*}{ Localidad } & \multirow{2}{*}{$\mathrm{CL}_{50}$} & \multirow{2}{*}{ IC $95 \%$} & \multirow{2}{*}{$\mathrm{CL}_{90}$} & \multirow{2}{*}{$\mathrm{IC}^{1} 95 \%$} & \multicolumn{3}{|c|}{ Pendiente } \\
\hline & & & & & \pm E.E. & $\mathrm{X}^{2}$ & g.1. \\
\hline Noaza & 519,14 a & $369,07-615,06$ & $922,14 b$ & $844,56-1.002,70$ & $5,14 \pm 0,94$ & 2,32 & 3 \\
\hline Parca & $862,40 \mathrm{a}$ & $508,85-1.017,70$ & $1.497,79 \mathrm{a}$ & $1.224,46-3.735,85$ & $5,35 \pm 0,61$ & 11,26 & 3 \\
\hline
\end{tabular}

1 Las concentraciones letales $\left(\mathrm{CL}_{50},{ }_{90}\right)$ seguidas de letras iguales no son significativamente diferentes, basado en que los IC al $95 \%$ se traslapan.

Análisis Probit (SPSS Statistics 21); CL: concentración letal; IC: intervalo de confianza; E.E.: error estándar; X²: chi cuadrado; g.1.: grados de libertad. 
como Rongai et al. (1998) no han detectado efectos del pirimicarb sobre el áfido A. gossypii incluso con las dosis máximas ensayadas $2.400 \mu \mathrm{m} \cdot \mathrm{L}^{-1}$. En cuanto a la relación entre la dosis recomendada para campo y la $\mathrm{CL}_{50}$, Desneux et al. (2004) obtuvieron un valor de $67,99 \mathrm{mg} \cdot \mathrm{L}^{-1}$ para pririmicarb aplicado contra $M$. persicae indicando una gran diferencia entre la toxicidad mostrada por este i.a. y la dosis recomendada por el fabricante. A pesar de los altos valores de la $\mathrm{CL}_{50}$ obtenidos en este estudio y que al compararlos con la literatura pueden considerarse como típicos de resistencia, nuestros resultados ubican a la $\mathrm{CL}_{50}$ dentro del rango de la dosis recomendada por el fabricante. Al respecto, Hugh et al. (2003) indican que $A$. gossypii muestra una alta susceptibilidad a imidacloprid mostrando una $\mathrm{CL}_{50}$ más baja que la dosis recomendada. Raboudi et al. (2012) encontraron que el porcentaje de las curvas de mortalidad mostraron un alto nivel de áfidos de la especie Macrosiphum euphorbiae (Thomas) (Hemiptera: Aphididae) resistentes a insecticidas del grupo de los carbamatos, donde, en todos los casos, la $\mathrm{CL}_{50}$ fue de más de $20 \mathrm{X}$ la concentración recomendada, lo que confirma la baja mortalidad. En cuanto a la proporción de resistencia, Furiatti y Lázarri (2000) encontraron valores entre 2,5X y 9,2X para adultos ápteros de $M$. persicae a pirimicarb.

\section{Conclusiones}

La dosis mínima recomendada por el fabricante (937,5 $\mathrm{mg} \cdot \mathrm{L}^{-1}$ de pirimicarb) produjo mortalidades entre $77,40 \%$ y $87,30 \%$ (Localidad de Noaza) y entre $40,40 \%$ y $42,60 \%$ (localidad de Parca) de áfidos adultos de $A$. craccivora. Para los áfidos de la localidad de Noaza (sin presión de selección por pirimicarb) las $\mathrm{CL}_{50,90}$ se ubican entre las dosis mínima y máxima recomendadas por el fabricante. En el caso de los áfidos provenientes de la localidad de Parca (con presión de selección), sólo la $\mathrm{CL}_{50}$ se encuentra en el rango recomendado. Para alcanzar la mortalidad de campo $\left(\mathrm{CL}_{90}\right)$, la concentración de pirimicarb requerida sobrepasa la dosis máxima recomendada. La proporción de resistencia (PR) fue de 1,7X indicando que aún no existen indicios de resistencia en los áfidos de la localidad de Parca, pudiendo ser controlados con este aficida, debiéndose respetar las dosis recomendadas y realizando rotación de insecticidas, para no acelerar el desarrollo de resistencia.

\section{Agradecimientos}

Los autores agradecen el apoyo financiero recibido de la Universidad Arturo Prat, Iquique, Chile.

\section{Literatura Citada}

Abbott, W.S.

1925. A method of computing the effectiveness of an insecticide. Journal of Economic Entomology, 18: 265-267.

Araya, M.; Araya, J.; Guerrero, M.

2004. Efectos de algunos insecticidas en dosis subletales sobre adultos de Aphidius ervi Haliday (Hymenoptera: Aphidiidae). Boletín de Sanidad Vegetal. Plagas, 30: 247-254.

Baker, R.T.

1978. Insecticide resistance in the green peach-potato aphid Myzus persicae (Sulz.) (Hemiptera: Aphididae). New Zealand Journal of Experimental Agriculture, 6 (1): 77-82. Bartlett, B.

1963. The contact toxicity of some pesticide residues to hymenopterous parasites and coccinellid predators. Journal of Economic Entomology, 56: 694-698.

Cerna, E.; Ochoa, Y.; Aguirre, A.; Badii, M.; Gallegos, G.; Landeros, J.

2009. Niveles de resistencia en poblaciones de Tetranychus urticae en el cultivo de la fresa. Revista Colombiana de Entomología, 35: 47-51.

De La Pava, N.; Sepúlveda-Cano, P.

2015. Biología del áfido negro (Aphis craccivora: Aphididae) sobre fríjol caupi (Vigna unguiculata, Fabaceae). Acta Biológica Colombiana, 20 (3): 93-97.
Desneux, N.; Rafalimanana, H.; Kaiser, L.

2004. Dose-response relationship in lethal and behavioural effects of different insecticides on the parasitic wasp Aphidius ervi. Chemosphere, 54: 619-627.

Fenoll, J.; Garrido, I.; Hellín, P.; Flores, P.; Vela, N.; Navarro, S. 2015. Photocatalytic oxidation of pirimicarb in aqueous slurries containing binary and ternary oxides of zinc and titanium. Journal of Photochemistry and Photobiology A: Chemistry, 298: 24-32.

Fuentes-Contreras, E.; Figueroa, Ch.C.; Silva, A.X.; Bacigalupe. L.D.; Briones, L.M.; Foster, S.P.; Unruh, T.R.

2013. Survey of resistance to four insecticides and their associated mechanisms in different genotypes of the green peach aphid (Hemiptera: Aphididae) from Chile. Journal of Economic Entomology, 106 (1): 400-407.

Furiatti, R.; Lázarri, S.

2000. Efeito da aplicacao de pirimicarbe sobre populacoes de campo de Myzus persicae (Sulz.) (Homoptera: Aphididae) com diferentes níveis de esterases. Anais da Sociedade Entomológica do Brasil, 29 (4): 739-747.

Herron, G.A.; Powis, K.; Rophail, J.

2001. Insecticide resistance in Aphis gossypii Glover (Hemiptera: Aphididae), a serious threat to Australian cotton. Australian Journal of Entomology, 40: 85-91. 
Hugh, E.; Conway, T.; Kring, J.; Ron, M.

2003. Effect of imidacloprid on wing formation in the cotton aphid (Homoptera: Aphididae), Florida entomologist, 86 (4): 474-476.

Iannacone, J.; Lamas, G.

2003. Efectos toxicológicos del nim, rotenona y cartap sobre tres micro-avispas parasitoides de plagas agrícolas en el Perú. Boletín de Sanidad Vegetal. Plagas, 29: 123-142.

Klein, C.; Waterhouse, D.F.

2000. The distribution and importance of arthropods associated with agriculture and forestry in Chile (Distribución e importancia de los artrópodos asociados a la agricultura y silvicultura en Chile). ACIAR Monograph 68. Canberra, Australia, 234 p.

Kraiss, H.; Cullen, E.M.

2008. Insect growth regulator effects of azadirachtin and neem oil on survivorship, development and fecundity of Aphis glycines (Hemiptera: Aphididae) and its predator, Harmonia axyridis (Coleoptera: Coccinellidae). Pest Management Science, 64 (6): 660-668.

Kwon, D.H.; Choi, B.R.; Lee, S.W.; Clark, J.M.; Lee, S.H.

2009. Characterization of carboxylesterase-mediated pirimicarb resistance in Myzus persicae. Pesticide Biochemistry and Physiology, 93: 120-126.

Lionetto, M.G.; Caricato, R.; Calisi, A.; Schettino, T.

2011. Acetylcholinesterase inhibition as a relevant biomarker in environmental monitoring: new insights and perspectives. En: Ecotoxicology Around the Globe. Visser, J.E (Ed.). Nova Science Publishers. pp. 87-116.

Milatovic, D.; Gupta, R.C.; Aschner, M.

2006. Anticholinesterase toxicity and oxidative stress. Science World Journal, 6: 295-310.

Muñoz-Viveros, L.; Itzhak, J.; Molist, P.; González-Sierra, S.;

Gonález, P.; Álvarez, R.

2014. Microscopic study of galls induced by three species of Geopemphigus (Hemiptera: Aphididae: Eriosomatinae) on Pistacia mexicana. Arthropod-Plant Interactions, 8: 531-538.

Raboudi, F.; Fattouch, S.; Makni, H.; Makni, M.

2012. Biochemical and molecular analysis of the pirimicarb effect on acethylcholinesterase resistance in Tunisian populations of potato aphid Macrosiphum euphorbiae (Hemiptera: Aphididae). Pesticide Biochemistry and Physiology, 104 (3): 261-266.

Rizk, M.; Darwish, Y.; Eraky, S.; Atta, A.

2014. Impact of the pirimicarb resistance on the ability of the cotton aphids, Aphis gossypii Glover (Homoptera: Aphididae) in transmitting plant viruses. Journal of Phytopathology and Pest Management, 1 (1): 16-21.

Rongai, D.; Cerato, C.; Martelli, R.; Ghedini, R.

1998. Aspects of insecticide resistance and reproductive biology of Aphis gossypii Glover on seed potatoes. Potato Research, 41: 21-37.

Tabacian, H.; Ravan, S.; Bandani, A.R.

2011. Susceptibilities of two populations of Aphis gossiper Glover to selected insecticides. African Journal of Biotechnology, 10 (4): 670-674.

Tello, V.; Díaz, L.; Sánchez, M.

2013. Side effects of the natural pesticide Spinosad (GF-120 Formulation) on Eretmocerus paulistus (Hymenoptera: Aphelinidae), a parasitoid of the whitefly Aleurothrixus floccosus (Hemiptera: Aleyrodidae), under laboratory conditions. Ciencia e Investigación Agraria, 40 (2): 407-417.

Walker, M.; Stufkens, M.; Wallace, A.

2007. Indirect non-target effects of insecticides on Tasmanian Brown lacewing (Micromus tasmaniae) from feeding on lettuce aphid (Nasonovia ribisnigri). Biological Control, 43 (1): 31-40. 